This item was submitted to Loughborough's Research Repository by the author.

Items in Figshare are protected by copyright, with all rights reserved, unless otherwise indicated.

\title{
Prediction of irradiation spectrum effects in pyrochlores
}

PLEASE CITE THE PUBLISHED VERSION

http://dx.doi.org/10.1007/s11837-014-1158-x

\section{PUBLISHER}

Springer / @ The Minerals, Metals \& Materials Society

\section{VERSION}

AM (Accepted Manuscript)

\section{PUBLISHER STATEMENT}

This work is made available according to the conditions of the Creative Commons Attribution-NonCommercialNoDerivatives 4.0 International (CC BY-NC-ND 4.0) licence. Full details of this licence are available at: https://creativecommons.org/licenses/by-nc-nd/4.0/

\section{LICENCE}

CC BY-NC-ND 4.0

\section{REPOSITORY RECORD}

Uberuaga, B.P., C. Jiang, C.R. Stanek, K.E. Sickafus, Chris Scott, and Roger Smith. 2019. "Prediction of Irradiation Spectrum Effects in Pyrochlores". figshare. https://hdl.handle.net/2134/16929. 


\title{
Prediction of irradiation spectrum effects in pyrochlores
}

\author{
B. P. Uberuaga, C. Jiang, and C. R. Stanek \\ Materials Science and Technology Division, Los Alamos National Laboratory, Los Alamos, NM 87545 \\ K. E. Sickafus \\ Department of Materials Science and Engineering, University of Tennessee, Knoxville, TN \\ C. Scott and R. Smith \\ Department of Mathematical Sciences, Loughborough University, Loughborough, Leicestershire LE11 $3 T U$, UK
}

\begin{abstract}
The formation energy of cation antisites in pyrochlores $\left(\mathrm{A}_{2} \mathrm{~B}_{2} \mathrm{O}_{7}\right)$ has been correlated with the susceptibility to amorphize under irradation. Thus, density functional theory (DFT) calculations of antisite energetics can provide insights into the radiation tolerance of pyrochlores. Here, we show that the formation energy of antisite pairs in titanate pyrochlores, as opposed to other families of pyrochlores $(\mathrm{A}=\mathrm{Zr}$, Hf, or $\mathrm{Sn})$, exhibit a strong dependence on the separation distance between the antisites. Classical molecular dynamics simulations of collision cascades in $\mathrm{Er}_{2} \mathrm{Ti}_{2} \mathrm{O}_{7}$ show that the average separation of antisite pairs is a function of the primary knock-on atom energy that creates the collision cascades. Together, these results suggest that the radiation tolerance of titanate pyrochlores may be sensitive to the irradiation conditions and might be controllable via the appropriate selection of ion beam parameters.
\end{abstract}

\section{INTRODUCTION}

Understanding how materials respond to extreme environments such as irradiation is crucial for predicting their performance. Much work has been done to understand the radiation tolerance of complex oxides, candidates for advanced nuclear fuels ${ }^{1}$ and nuclear waste forms ${ }^{2}$. This is especially true of pyrochlores $\mathrm{A}_{2} \mathrm{~B}_{2} \mathrm{O}_{7}$, a prototypical complex oxide with a natural analogue. While the overall radiation tolerance of pyrochlores has been studied as a function of composition ${ }^{3}$, less attention has been devoted to how the radiation tolerance depends on the details of the irradiation spectrum. In this paper, using a combination of density functional theory (DFT) and molecular dynamics (MD) with empirical potentials, we examine how the radiation tolerance of pyrochlores may potentially depend on the irradiation spectrum.

Past work has suggested a clear relationship between the propensity of complex oxides to disorder and their radiation tolerance ${ }^{4,5}$. This concept is formulated in the following defect reaction pair:

$$
\begin{aligned}
A_{A}+B_{B} & \rightarrow A_{B}+B_{A} \\
O_{O} & \rightarrow V_{O}+O_{i} .
\end{aligned}
$$

These equations describe the formation of cation antisites and anion Frenkel pairs, defect reactions that occur when a material is irradiated. Exactly how these defects might be formed is not entirely clear, but a combined experimental-theoretical study suggests that the defects formed in pyrochlore under irradiation are antisites and that these must be formed directly in cascades, as the thermal evolution of cation defects is very slow ${ }^{6}$. Further, the accumulation of these defects also represents the transition for an ordered pyrochlore to a disordered fluorite, a phase transformation that is typically observed in irradiation experiments.
Past calculations, with empirical potentials ${ }^{4}$ and $\mathrm{DFT}^{7}$, have demonstrated that both individual disordering defects - isolated cation antisites and anion Frenkel pairs - and the total disorder of the pyrochlore structure correlate well with experimental observations of radiation tolerance. For example, both types of calculations show a peak in the titanate family of pyrochlores at $\mathrm{Gd}_{2} \mathrm{Ti}_{2} \mathrm{O}_{7}$, consistent with experimental measurements of the critical temperature for amorphization ${ }^{8}$. Further, both DFT and empirical potentials show that the energetics of the reactions represented in Eqs. 1 and 2 are much higher for titanates than for zirconates. However, none of these calculations have attempted to distinguish how the different pyrochlore compounds might react differently to different irradiation spectra. That is, are the energetics of the reactions in Eqs. 1 and 2 dependent on how the defects are created?

In this work, we examine the stability of antisite pairs in a series of pyrochlore compositions as a function of the antisite pair separation using DFT. We find that, while in most pyrochlores, there is little sensitivity of the antisite energy to their separation, this is not true for the titanates. We then perform MD simulations of collision cascades in pyrochlore to understand how the antisite distribution depends on the irradiation conditions. Together, these results suggest that the antisite separation could in principle be controlled via the irradiation spectrum and that the radiation tolerance of titanate pyrochlore, at least in early times, would depend on this spectrum effect.

\section{METHODOLOGY}

We use DFT to examine the energetics of antisite pairs in a series of pyrochlores. Our calculations are performed 
using the all-electron projector-augmented wave method within the generalized gradient approximation (GGA), as implemented in Vienna ab initio simulation package $(\text { VASP })^{9}$. The lattice parameters and internal atomic positions of all structures are fully relaxed using conjugategradients. As described in our previous work ${ }^{7}$, a planewave cutoff energy of $400 \mathrm{eV}$ and a $2 \times 2 \times 2 \mathrm{k}$-point sampling give fully converged results. The special quasirandom structure (SQS ${ }^{10}$ approach was used to model the fully disordered pyrochlore. Those results have been reported previously ${ }^{7}$ and are reproduced here for comparison.

Collision cascades were modeled in $\mathrm{Er}_{2} \mathrm{Ti}_{2} \mathrm{O}_{7}$, chosen as a typical titanate pyrochlore, using MD. The potential included a very-short range ZBL interaction ${ }^{11}$, an intermediate range Buckingham potential, and long-range electrostatic forces, calculated using the DPMTA (multipole) method ${ }^{12}$. The Buckingham parameters were taken from Ref. ${ }^{13}$. Fixed boundary conditions were used, which necessitated the adjustment of charges on the surface of the simulation block to avoid surface dipoles. Primary knock-on atom (PKA) energies from 1 to $6 \mathrm{keV}$ were simulated and the corresponding system sizes ranged from 155,593 to 948,773 atoms, respectively. Simulations were run for 4-6 ps, long enough for the thermal spike to settle and a (meta)stable defect structure to establish itself. Simulations were performed using each species as the PKA. The total number of simulations at each energy and for each PKA species is summarized in Table I.

\begin{tabular}{l|ccccc} 
& \multicolumn{4}{|c}{ PKA } & energy \\
PKA species & 1 & 2 & 4 & 5 & 6 \\
\hline $\mathrm{Er}$ & 14 & 11 & 6 & 5 & 3 \\
$\mathrm{O}$ & 14 & 11 & 4 & 4 & 3 \\
$\mathrm{Ti}$ & 14 & 11 & 6 & 5 & 3
\end{tabular}

TABLE I: Summary of the number of simulations performed at each PKA energy for each PKA species.

\section{RESULTS}

\section{A. DFT calculations of antisite energetics}

As described in a previous paper ${ }^{7}$, the energy calculated by DFT for complete disordering of the pyrochlore structure tends to increase as the B cation is changed from $\mathrm{Zr}$ to $\mathrm{Hf}$ to $\mathrm{Ti}$ to $\mathrm{Sn}$, again consistent with empirical calculations which examined the smallest clusters of disordering defects $\left(A_{B}+B_{A}+V_{O}+O_{i}\right)^{4}$. In these clusters, the cation antisite pair were created as nearest neighbors. Using DFT, we have calculated the energy of formation of a cation antisite pair in pyrochlore as a function separation of the pair. The results are shown in Figure 1. When the antisites are nearest neighbors, as in Fig. 1a, the formation energy of these individual defects generally follows the same trend as for overall disorder
(Fig. 1b), except that the titanate series appears slightly more accomodating of these types of defects than it is for full disorder. That is, when compared to e.g. the Hf series, the formation of nearest neighbor antisites is easier than the creation of a fully disordered pyrochlore. Further, when considering either nearest neighbor antisites or fully disordered pyrochlore, the titanate series is intermediate in stability, with all chemistries exhbiting lower energies for either type of disorder than the stannate series. When compared to the zirconate or hafnate families, the titanates exhibit both higher energies, for small cations, and lower energies, for large cations. This is a consequence of the fact that, as compared to the $\mathrm{Zr}$ or Hf families of pyrochlores, which display nearly linear trends in the disordering energies as a function of the $\mathrm{A}^{3+}$ cation radius, the titanate family exhibits a more complex behavior. As noted above, the titanate disordering energy is non-monotonic with $\mathrm{A}^{3+}$ cation radius, peaking at $\mathrm{A}=\mathrm{Gd}$, in accordance with experimental results ${ }^{8}$.

The opposite behavior is observed if the antisites are created far apart, as shown in Fig. 1c. In this case, while for the zirconates, hafnates, and stanates there is no appreciable difference in the antisite pair formation energy as a function of separation, for the titanates there is a great change in the formation energy of the antisite pair. The formation energy increases by at least $1 \mathrm{eV}$ for those titanates with a small A cation, and even more for those with a large A cation. The difference is most dramatic for $\mathrm{Nd}_{2} \mathrm{Ti}_{2} \mathrm{O}_{7}$ : when the antisites are created as nearest neighbors, this pyrochlore with $\mathrm{A}=\mathrm{Nd}$ has one of the lowest formation energies to create antisites. However, when the antisites are separated, it has one of the highest antisite formation energies. As a consequence, the relative ordering of the antisite stability in the different pyrochlore families shifts. While for nearest neighbor antisites, the titanate family resides intermediate between $\mathrm{Zr} / \mathrm{Hf}$ and $\mathrm{Sn}$, for separated antisites, the formation energy of antisites is greatest in the titanate family, even greater than the stannate family.

Following the hypothesis that the tendency for disorder is an indicator of radiation tolerance, this result suggests that the radiation tolerance of the titanate pyrochlores may be very sensitive to how the antisites are created. If they are created as nearest neighbors, they are relatively easy to form and the material may be relatively radiation tolerant. If they are created far apart, the energetic cost is much greater and the material will respond relatively poorly to irradiation. In particular, the radiation stability of the titanates relative to the stannates would depend on the how the antisites are created.

\section{B. MD simulations of collision cascades}

As discussed above, the formation energy of antisite pairs in titanate pyrochlores is very sensitive to the distance separating those pairs. This separation, in turn, is a function of the irradiation spectrum. We have per- 

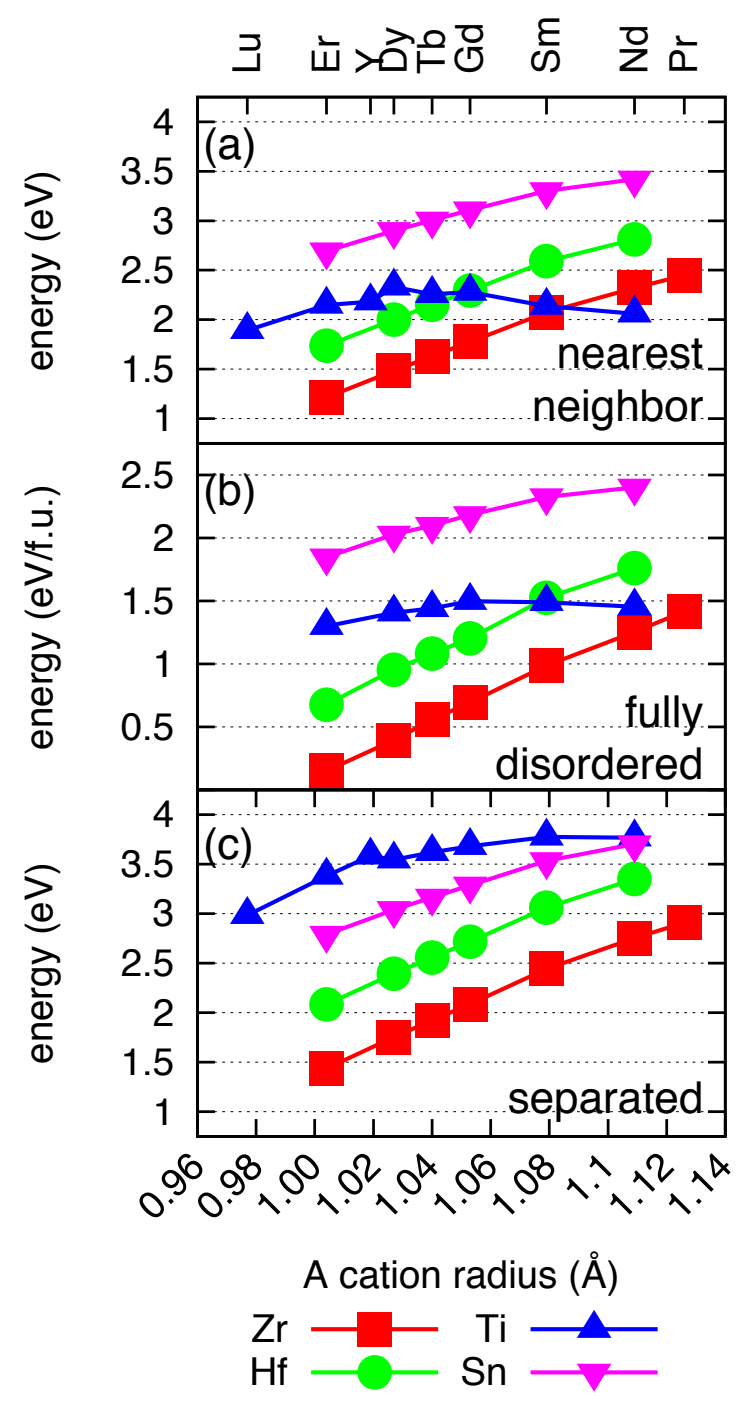

FIG. 1: Formation energy of cation antisite pairs in four families of pyrochlores as a function of the antisite separation distance. (a) Formation energy when the antisites are separated. (b) Formation energy when the antisites are nearest neighbors. (c) Formation energy of fully disordered pyrochlore, as compared to ordered pyrochlore, in $\mathrm{eV}$ per formula unit (f.u.) of pyrochlore. The titanates in particular are very sensitive to the separation distance. The lines are guides for the eye.

formed MD simulations of defect production in $\mathrm{Er}_{2} \mathrm{Ti}_{2} \mathrm{O}_{7}$ to determine how antisite separation depends on the irradiation conditions. We have simulated collision cascades with primary knock-on atom (PKA) energies varying from 1 to $6 \mathrm{keV}$. The mean separation of antisites was calculated once the thermal spike of the cascade dissipated, and the results are shown in Fig. 2. There is a clear dependence of the antisite separation on PKA energy, regardless of the PKA species. In fact, over this energy range, the dependence is roughly linear, at least for $\mathrm{O}$ and Ti PKAs, though the slope of that dependence does depend on PKA species. For cases when the PKA species is Er, there is not such a strong dependence of the antisite separation with PKA energy, though there is still more separation for larger PKAs than for smaller PKAs.

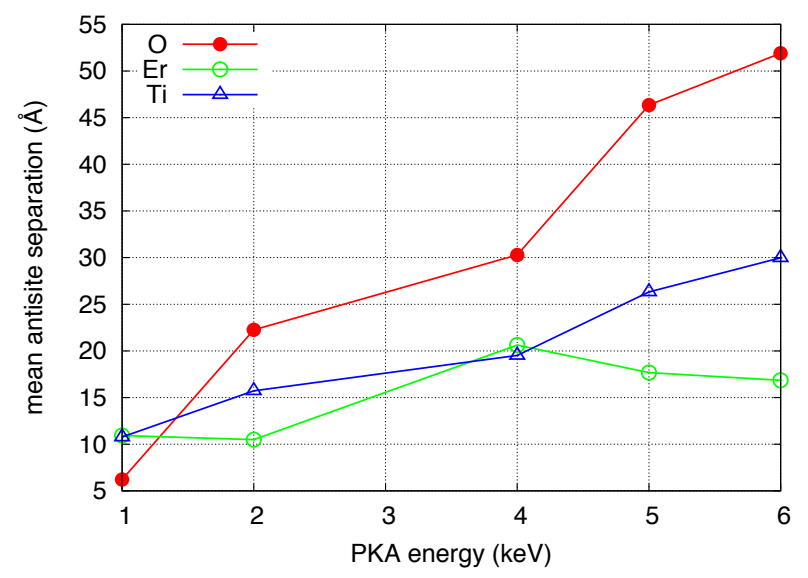

FIG. 2: Average separation of antisites as a function of PKA energy per cascade, for each PKA species. The lines are guides for the eye.

Figure 3 shows the distribution of antisite separations for $6 \mathrm{keV}$ PKAs. For both $\mathrm{Ti}$ and Er PKAs, there is simple Poisson distribution of separations. However, in the case of an O PKA, the antisite separations show a bimodal distribution, with both relatively close and far antisites and an intermediate region where very few antisites are created. This is a signature of subcascade branching. The O PKA tends to channel quite far and can thus induce secondary cascades far from the site of the first cascade. This results in two (or more) regions of where antisites are formed in the material.

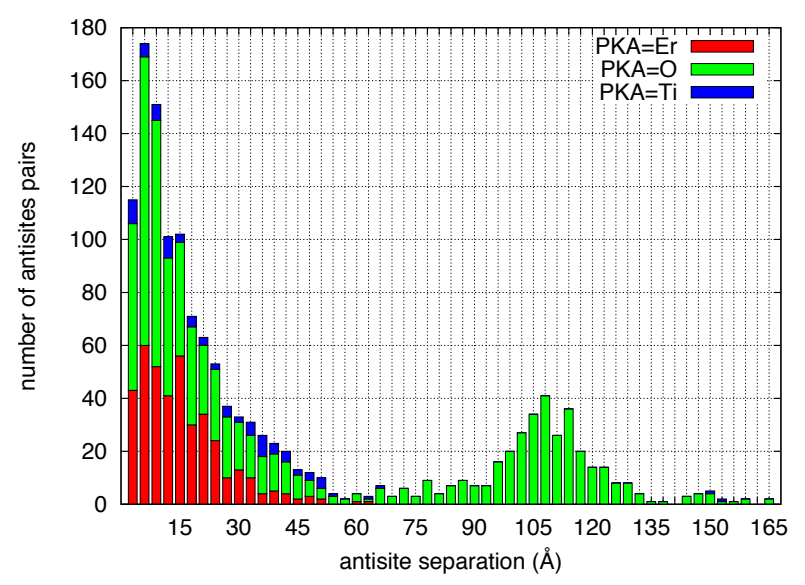

FIG. 3: Distribution of the number of antisite pairs as a function of their separation. The distribution is broken down into contributions from simulations in which Er, O, and Ti were the PKA. Each bar represents a 3 Åbin of distances. 
Thus, in the titanates, the formation energy of antisites depends on their separation and that separation, in turn, depends on the irradiation conditions. While, eventually, as disorder accumulates in the material, the properties should tend towards those predicted by calculations of fully disordered pyrochlore, these results represent the initial build up towards full disorder and will determine the kinetic pathways by which full disorder is achieved. As disorder accumulates, the titanates will eventually begin to amorphize as the cost of creating that disorder will be too great. However, for low doses or during the initial stages of damage, the titanates may respond better than even the zirconates.

We note that, at higher temperatures at which thermally activated events involving cation interstitials and vacancies can occur, the evolution of those defects can lead to the formation of antisites. Ti interstitials and vacancies are particularly prone to such events, forming A cation antisites and A cation interstitials and vacancies, respectively ${ }^{6}$. Depending on the irradiation temperature, one could control the antisite distribution rather significantly simply by activating such processes.

\section{DISCUSSION AND CONCLUSIONS}

The PKA energies simulated here are typical of the end-of-range secondary knock-on energies that occur in the ballistic damage regime in real irradiation experiments. Thus, by controlling the irradiation spectrum, the observed radiation tolerance of titanates may also be controlled, at least initially. For example, electron irradiation tends to produce isolated defects. If the irradiation energy is just above the displacement threshold energy, only very near antisite defects will be created. If, however, higher energy electrons or light ions are used, defects will be created further apart. This is particularly true of oxygen. If electron irradiation is used at an energy in which oxygen is displaced but not the cations, large antisite separations could form and the material might be more susceptible to amorphization than in the case of heavy ion damage which creates PKAs on all sublattices. Thus, the observed radiation tolerance may depend on the detailed irradiation conditions.

Of course, as the irradiation progresses and the concentration of antisites accumulates, the system will more closely resemble the fully disordered state. That is, ultimately, the behavior of the material will be governed by the energetics of nearest neighbor antisites as these will predominate the material. However, at early times, when the antisite concentration is relatively small, the energetics will be dependent on separation and thus the irradiation conditions. It is possible that one can choose irradiation conditions in which the relative tolerance of titanates versus stannates can be swapped, based on the energetics presented in Figs. 1a-c.

Other factors related to the irradiation spectrum may also be important. It is well established that electronic effects, introduced when the ion energies are relatively high, can significantly alter the amorphization susceptibility of complex oxides ${ }^{14}$. Further, in perovskites, it has been demonstrated that while heavy ions such as Xe can quickly amorphize the material even at room temperature, irradiations with lighter Ne ions do not induce amorphization by similar doses even at $20 \mathrm{~K}^{15}$. Thus, a number of competing factors must be accounted for to fully understand and predict the response of a complex oxide to irradiation.

To conclude, given that amorphization resistance is intimately linked to the ability of a material to accomodate disorder, the radiation tolerance of titanates, especially relative to other pyrochlores, may depend sensitively on the irradiation conditions. The ability of titanates to accomodate disorder depends on just how that disorder is introduced, much more so than in the other pyrochlore families studied here. Thus, while titanate pyrochlores may exhibit poor radiation tolerance in some situations, in others they may exhibit relatively robust behavior. These results demonstrate the sensitivity of material behavior to details of the environmental conditions and illustrates the challenges in developing a complete understanding of how materials behave in extreme environments.

\section{ACKNOWLEDGMENTS}

This work was supported by the U.S. Department of Energy, Office of Science, Basic Energy Sciences, Materials Sciences and Engineering Division. Los Alamos National Laboratory is operated by Los Alamos National Security, LLC, for the National Nuclear Security Administration of the (U.S.) Department of Energy under contract DE-AC52-06NA25396. We thank Arthur F. Voter for helpful discussions.
1 D. Olander, J. Nucl. Mater. 389, 1 (2009).

2 W. J. Weber, C. R. A. Catlow, L. W. Hobbs, H. Matzke, M. Nastasi, and E. R. Vance, J. Mater. Res. 13, 1434 (1998).

3 M. Lang, J. Lian, J. Zhang, F. Zhang, W. J. Weber, C. Trautmann, and R. C. Ewing, Phys. Rev. B 79, 224105 (2009).
${ }^{4}$ K. E. Sickafus, L. Minervini, R. W. Grimes, J. A. Valdez, M. Ishimaru, F. Li, K. J. McClellan, and T. Hartmann, Science 289, 748 (2000).

${ }^{5}$ K. E. Sickafus, R. W. Grimes, J. A. Valdez, A. Cleave, M. Tang, M. Ishimaru, S. M. Corish, C. R. Stanek, and B. P. Uberuaga, Nat. Mater. 6, 217 (2007).

6 Y. H. Li, B. P. Uberuaga, C. Jiang, S. Choudhury, J. A. 
Valdez, M. K. Patel, J. Won, Y. Q. Wang, M. Tang, D. J. Safarik, et al., Phys. Rev. Lett. 108, 195504 (2012).

7 C. Jiang, C. R. Stanek, K. E. Sickafus, and B. P. Uberuaga, Phys. Rev. B 79, 104203 (2009).

8 J. Lian, J. Chen, L. M. Wang, R. C. Ewing, J. M. Farmer, L. A. Boatner, and K. B. Helean, Phys. Rev. B 68, 134107 (2003).

9 G. Kresse and J. Furthmuller, Phys. Rev. B 54, 11169 (1996).

10 A. Zunger, S. H. Wei, L. G. Ferreira, and J. E. Bernard, Phys. Rev. Lett. 65, 353 (1990).

11 J. Ziegler, J. Biersack, and U. Littmark, The Stopping and
Range of Ions in Matter (Pergamon, New York, 1985).

12 W. T. Rankin and J. A. B. Jr., Proceedings of the 1995 IEEE Symposium on High Performance Distributed Computing (IEEE Computer Society Press, Los Alamitos, California, 1995), p. 17.

${ }^{13}$ L. Minervini, R. W. Grimes, and K. E. Sickafus, J. Am. Ceram. Soc. 83, 1873 (2000).

14 S. J. Zinkle, V. A. Skuratov, and D. T. Hoelzer, Nucl. Instr. and Meth. B 191, 758 (2002).

15 A. Meldrum, L. A. Boatner, W. J. Weber, and R. C. Ewing, J. Nucl. Mater. 300, 242 (2002). 